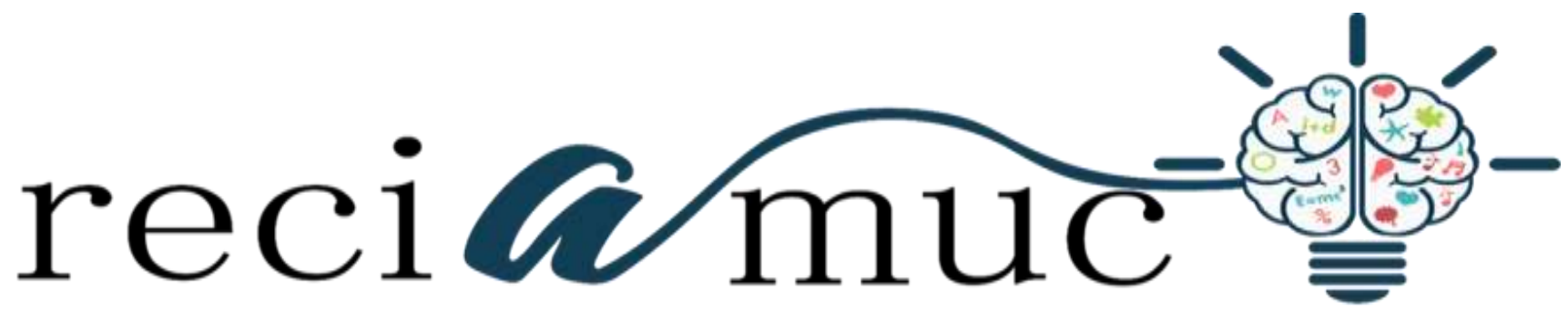

Revista cientifica de investigación actualización del mundo de las ciencias

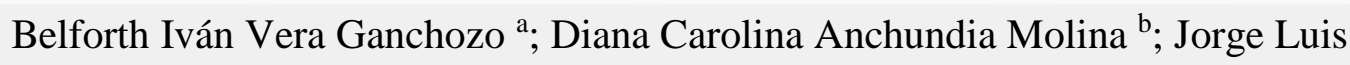

Castillo Avendaño ${ }^{c}$; Edith Elizabeth Cedeño Gavilánez ${ }^{\mathrm{d}}$;

María José Saltos Loor ${ }^{\mathrm{e}}$; Dolly Natasha Ross Mera ${ }^{\mathrm{f}}$

Procedimientos Adecuados para los Pacientes Intervenidos en Cirugía de Reasignación sexual

Procedures Suitable for the Patients who Underwent Sex Reassignment Surgery In

Revista Científica de Investigación actualización del mundo de las Ciencias. Vol.

3 núm., 2, abril, ISSN: 2588-0748, 2018, pp. 976-995

DOI: $10.26820 /$ reciamuc/3.(2).abril.2019.976-995

URL: http://reciamuc.com/index.php/RECIAMUC/article/view/375

Código UNESCO: 3205 Medicina Interna

Tipo de Investigación: Artículo de Revisión

(C) RECIAMUC; Editorial Saberes del Conocimiento, 2019

Recibido: $15 / 01 / 2019$

Aceptado: 07/02/2019

Publicado: 01/04/2019

Correspondencia: belforth15@hotmail.com

a. Médico Cirujano; Médico Residente UCI - Hospital Verdi Cevallos Balda; Portoviejo - Ecuador; belforth15@hotmail.com

b. Médico Cirujano; Médico en Función Hospitalaria - Hospital Dr. Gustavo Domínguez; Santo Domingo Ecuador; dcamolina@ hotmail.com

c. Médico Cirujano; Médico General asistencial en funciones hospitalarias - Hospital Dr. Gustavo Domínguez; Santo Domingo - Ecuador; ballack1306@ hotmail.com

d. Médico Cirujano; Médico General en Funciones Hospitalarias - Hospital Dr. Gustavo Domínguez; Santo Domingo - Ecuador; edith-eliza@ hotmail.com

e. Médica Cirujana; Médico General Asistencial - Hospital Verdi Cevallos Balda; Portoviejo - Ecuador; maj_o_sl@hotmail.com

f. Médico Cirujano; Médico residente de UCI - Clínica del Sol Manta, Médico ocupacional - Consorcio RR\&O; Portoviejo-Ecuador; naty-ross@ hotmail.com 


\section{Procedimientos Adecuados para los Pacientes Intervenidos en Cirugía de \\ Reasignación sexual}

Vol. 3, núm. 2., (2019)

Belforth Iván Vera Ganchozo; Diana Carolina Anchundia Molina; Jorge Luis Castillo Avendaño;

Edith Elizabeth Cedeño Gavilánez; María José Saltos Loor; Dolly Natasha Ross Mera

\section{RESUMEN}

Los conceptos sexo y género, confluyen simultáneamente en un individuo que biológicamente posee un cuerpo sexuado para clasificarlo como hombre o mujer, caracterizado por conductas culturalmente masculinas o femeninas respectivamente. Sin embargo, de quien se enamore esa persona viene a ser el factor determinante para su genitalidad acorde con el género con el cual se siente identificado, esto, abre el camino hacia un nuevo concepto la transexualidad, caracterizada por tener un cerebro diferente al sexo biológico que posee el individuo; además, pasan por un malestar persistente al no sentirse de acuerdo con su género físico, también experimentan confusión de su propia auto identidad. Ante esta realidad, las ciencias médicas registran nuevas investigaciones con el fin de ofrecer a ese sujeto la oportunidad de lograr mediante una cirugía reconocida como: reasignación sexual, para así implicar intervenciones, que hacen una pausa necesaria en el caso de niñas, niños, adolescentes y adultos transexuales e intersexuales a reconocer los derechos específicos que les son inherentes. Por ello, para su realización, es importante que el paciente sea evaluado por varios especialistas tales como: sexólogo, endocrinólogo, psiquiatra, cirujano plástico, todos ellos serán los encargados de coordinar el tratamiento previo para establecer de manera conjunta el mejor momento para la realización de la cirugía: La actuación de este grupo multidisciplinario, dará los procedimientos a seguir una vez intervenido entre ellos: reposo, control con el psicólogo, utilización de medicamentos para reducir los efectos del dolor y procesos de coagulación cuando se amerite entre otros. Consideraciones, importantes para la presentación de este artículo cuyo objetivo fue analizar los procedimientos adecuados para los pacientes intervenidos en cirugías de reasignación sexual. El mismo corresponde a una investigación documental aspecto que hizo posible la construcción de diferentes argumentaciones como conclusiones.

Palabras Claves: Procedimientos adecuados en pacientes intervenidos; Cirugías de reasignación sexual. 


\title{
Procedimientos Adecuados para los Pacientes Intervenidos en Cirugía de Reasignación sexual
}

Vol. 3, núm. 2., (2019)

Belforth Iván Vera Ganchozo; Diana Carolina Anchundia Molina; Jorge Luis Castillo Avendaño; Edith Elizabeth Cedeño Gavilánez; María José Saltos Loor; Dolly Natasha Ross Mera

\begin{abstract}
Concepts sex and gender converge simultaneously in an individual who has biologically a sexed body to classify it as male or female, characterized by conduct culturally male or female respectively. However, who fell in love with that person comes to be the determining factor for their sexuality according to the gender with which you feel identified, this opens the way towards a new concept trans sexuality, characterized by having a different sexual brain biological that has the individual; In addition, pass through a malaise persistent not to feel according to your physical gender, also experience your own self-identity confusion. Faced with this reality, the medical sciences recorded new research in order to offer the opportunity to achieve a recognized surgical that subject as: sex reassignment, to thus involve interventions that make a necessary pause in the case of children, adolescents and adults transsexuals and intersexuals to recognize the specific rights that are inherent to them. Therefore, for its realization, it is important that the patient be evaluated by several specialists such as: endocrinologist, sexologist, psychiatrist, plastic surgeon, all of them will be responsible for coordinating pretreatment to establish way Joint the best time to perform the surgery: the performance of this multidisciplinary group, will give the procedures to follow once intervened between them: rest, control with the psychologist, the use of drugs to reduce the effects of the pain and processes of coagulation when it warrants among others. Important considerations for the presentation of this article whose aim was to analyze the procedures suitable for the patients who underwent sex reassignment surgery. It corresponds to a documentary research aspect that made possible the construction of different arguments as conclusions.
\end{abstract}

Key Words: Proper procedures in sex reassignment surgery; Surgery patients. 


\section{Procedimientos Adecuados para los Pacientes Intervenidos en Cirugía de \\ Reasignación sexual}

Vol. 3, núm. 2., (2019)

Belforth Iván Vera Ganchozo; Diana Carolina Anchundia Molina; Jorge Luis Castillo Avendaño;

Edith Elizabeth Cedeño Gavilánez; María José Saltos Loor; Dolly Natasha Ross Mera

\section{Introducción.}

La humanidad desde sus inicios, se encuentra caracterizada por una división sexista que visualiza dos géneros femenino y masculino, aceptado socialmente en el mundo. Pero a lo largo de la historia, nacen personas con comportamientos diferentes en relación a su sexo biológico, aspecto de significación para las investigaciones científicas dentro del campo médico y demás disciplinas. Estos procesos dieron cabida a la presencia de un nuevo término en la clasificación de los individuos la transexualidad vista como una manera antagónica presente en los individuos que poseen órganos sexuales externos e internos relacionados con un género, pero sus actitudes son distantes y por ende actúan de manera contraria a los patrones culturales preestablecidos.

En razón de lo anterior, se puede entender que el surgimiento y aceptación de la transexualidad hoy en día se da en personas cuyo sexo biológico difiere de aquel que experimenta o siente como propio; por lo tanto, hay dos tipos de transexualidad: femenina y masculina. Por ello, se visualiza como un severo trastorno físico en el que una persona, varón o mujer, se ve condenado a crecer (por un mecanismo desconocido en la embriogénesis) en un cuerpo contrario al de su género real. Está en un cuerpo que no le toca. De allí, que hay que existen muchos tipos de sexo entre ellos: el cromosómico, gonadal, hormonal, genital interno, genital externo y finalmente cerebral. $\mathrm{Al}$ conocer esto es más fácil valorar lo que puede pasar y se produce un error en la diferenciación de alguno o varios de estos sexos. Afortunadamente la ciencia está avanzando en el conocimiento del trastorno y de esta manera se puede ofrecer nuevas alternativas que ayudan a estos individuos a tener calidad de vida.

De lo antes expuesto, se precisa que, en el campo de las ciencias médicas, se busca lograr el respectivo abordaje de la realidad que caracteriza a los transexuales, razón por la cual, se introduce enfoques y tratamientos multidisciplinarios, que se inician con un asesoramiento y tratamiento por expertos psiquiatras y psicólogos, que den un diagnóstico fiable de la transexualidad. Aunado a la presencia de un equipo de endocrinólogos encargados de llevar una correcta y controlada terapia hormonal substitutoria. Asimismo, la utilización de un test de vida real. Solo así se puede plantear una intervención quirúrgica que les ayude a lograr la respectiva 


\section{Procedimientos Adecuados para los Pacientes Intervenidos en Cirugía de Reasignación sexual}

Vol. 3, núm. 2., (2019)

Belforth Iván Vera Ganchozo; Diana Carolina Anchundia Molina; Jorge Luis Castillo Avendaño; Edith Elizabeth Cedeño Gavilánez; María José Saltos Loor; Dolly Natasha Ross Mera

reasignación sexual y con ello garantizarle mediante técnicas apropiadas su actuación en la sociedad de manera cónsona a sus propias necesidades como sujeto que se circunscribe a un entorno determinado.

Ante este planteamiento, la medicina posee hoy en día una intervención quirúrgica reconocida como reorganización sexual, basada en una serie de procedimientos que modifican los genitales por nacimiento de una persona con el fin de que el paciente tenga el género con el cual se identifica. En esta dirección, Rizo (2015) indica “esta cirugía suele realizarse a personas transexuales que optan a ella como parte de su cambio de sexo, intersexualidad especialmente en la infancia, el proceso se conoce como: reasignación sexual, cirugía de reasignación genital, entre otros términos médicos" (p.28). Es decir, la práctica quirúrgica de este proceso médico, hace posible lograr un cambio a través de la genitoplastía feminizante o penectomía, orquiectomía y vaginoplastía, que en el ámbito clínico se utilizan para las mujeres y en los hombres transexuales, se utiliza genitoplastía masculinizante, metoidioplastía o faloplastia.

Cabe agregar, que las personas que se someten a la cirugía de reasignación sexual se les conoce comúnmente como transexuales: (trans) por haber sido sometidas a un cambio, una transformación, un paso; (sexuales) debido a las características propias que poseen (no acciones sexuales) de una persona. Más recientemente, estas personas se identifican como transgénico y no como transexuales. Desde esta perspectiva, se entiende que dicha transición según Ruiz (2015) "es un proceso mediante el cual cambian su aspecto para convertirse en una persona del género que se siente internamente". (p.14). Es decir, significa la inclusión de un tratamiento médico y hormas que propician cambios físicos externos, además, de modificar su apariencia y vestimenta, lo que asegura declararse abiertamente ante sus grupos y familia como un transexual, proceso que puede abarcar un largo y continuo tiempo.

Sin embargo, a pesar de los avances médicos en materia de la reasignación sexual, se presentan consecuencias negativas en los pacientes, debido a los cambios físicos, adaptación a un nuevo esquema de vida, reinserción a sus actividades laborales, sociales y familiares, tal como lo 


\section{Procedimientos Adecuados para los Pacientes Intervenidos en Cirugía de \\ Reasignación sexual}

Vol. 3, núm. 2., (2019)

Belforth Iván Vera Ganchozo; Diana Carolina Anchundia Molina; Jorge Luis Castillo Avendaño;

Edith Elizabeth Cedeño Gavilánez; María José Saltos Loor; Dolly Natasha Ross Mera

asevera Ruiz (ob.cit) "después del cambio de sexo, las personas con transexualismo tienen riesgos notablemente más altos de mortalidad conducta suicida". (p.16). Lo planteado, lleva a destacar que, los procesos quirúrgicos de reasignación sexual, debe ser debidamente canalizados a través de psiquiatras y psicólogos, además, de lograr el respectivo acompañamiento familiar como medidas que ayuden al paciente a manejar adecuadamente los cambios físicos observados y desde esta acción mejorar sus niveles de aceptación.

Asimismo, este último autor destaca que después del cambio de sexo, en las personas transgénero tienen riesgos:

Notablemente más altos de mortalidad, conducta suicida, patología psiquiátrica que el conjunto de la población ubicado en un $41 \%$ porcentaje más alto que el 4,6\% del conjunto de la población de Estados Unidos, en la población gay se sitúa entre 10 y 20\%. Además, el cambio de sexo, aunque alivia la disforia de género, puede ser un tratamiento insuficiente para el transexualismo, por lo cual, debe llevar a mejorar la atención psiquiátrica y médica a este grupo de pacientes. (p. 16)

Por lo tanto, una vez realizado este procedimiento quirúrgico, es importante que el equipo multidisciplinario ofrezca al paciente una serie de recomendaciones pertinentes para lograr su inserción efectiva en la sociedad, pues, no todas las personas lo hacen de la misma manera. Algunas realizan una transición social y no médica. Otras pueden hacer una transición médica mediante solo uno o algunos de los procedimientos establecidos. Hay personas que toman hormonas y optan por evitar las cirugías, o simplemente eligen un solo tipo de cirugía.

En consecuencia, la decisión que el transexual asuma con respecto a la realización de una intervención quirúrgica de reasignación sexual, se encuentra íntimamente relacionada con la disforia de género, que implica la existencia de una discrepancia significativa entre el sexo anatómico y la percepción que tiene de sí mismo, como masculino, femenino, mixto, neutro u otra identidad sexual. Este sentimiento hace que la persona experimente una angustia significativa, lo que menoscaba su capacidad para desenvolverse, siendo la transexualidad una forma extrema de la disforia de género. Por ello, cuando se cumple el respectivo proceso 


\section{Procedimientos Adecuados para los Pacientes Intervenidos en Cirugía de Reasignación sexual}

Vol. 3, núm. 2., (2019)

Belforth Iván Vera Ganchozo; Diana Carolina Anchundia Molina; Jorge Luis Castillo Avendaño; Edith Elizabeth Cedeño Gavilánez; María José Saltos Loor; Dolly Natasha Ross Mera

quirúrgico de reasignación sexual, le compete al equipo multidisciplinario seleccionar una gama de procedimientos adecuados, que están estrechamente relacionados con las características psicológicas del paciente, aunado a las medidas de control médico en materia del tratamiento, vigilancia médica, monitoreo de los cambios en materia conductual especialmente en aquellos relacionados con la confirmación de su percepción externa con respecto a su identidad sexual.

En consecuencia, estas apreciaciones desarrolladas a lo largo del proceso investigativo, hacen posible entender el significado que posee para un individuo transexual, su cambio de sexo, mediante la realización de una intervención quirúrgica que le ayude a lograr la respectiva modificación perceptiva de su identidad sexual, por ello, los avances en materia médica a través de la reasignación sexual, se convierte en una acción efectiva para este grupo de individuos, pues, tienen oportunidad de vivir conforme a los cambios registrados, de allí, la necesidad de ofrecer un tratamiento multidisciplinario e integrado de transexualidad, dando las máximas garantías médicas para la salud del paciente. De allí, que el equipo médico lo constituyen profesionales altamente cualificados y especializados en los diversos campos de la cirugía de reasignación sexual: cirujano plástico estético y reparador, ginecólogos y cirujanos del aparato digestivo entre otros.

Aspectos, que sirven de soporte referencial para la realización y presentación del artículo, mediante el cual se busca llegar a un análisis relacionado con los procedimientos adecuados para los pacientes intervenidos en cirugía de reasignación sexual, por lo cual, es importante fijar una posición clara en cuanto al método a emplear, tipo de investigación, fuentes y técnicas de recolección de información, eventos fundamentales para lograr la respectiva construcción del escrito bajo lineamientos realmente científicos y ajustados a las normas de redacción.

\section{Método.}

El fenómeno que sirve de referencia para la realización de un proceso investigativo, debe ser debidamente operacionalizado con el fin de proceder a interpretar el problema. Al respecto, Martínez (2018) indica que el "el trabajo de investigación es una experiencia significativa de situaciones reales que sirven para el análisis de la acción a realizar”. (p.26). De allí, que cada una 


\section{Procedimientos Adecuados para los Pacientes Intervenidos en Cirugía de \\ Reasignación sexual}

Vol. 3, núm. 2., (2019)

Belforth Iván Vera Ganchozo; Diana Carolina Anchundia Molina; Jorge Luis Castillo Avendaño;

Edith Elizabeth Cedeño Gavilánez; María José Saltos Loor; Dolly Natasha Ross Mera

de las actividades propuestas, deben ser debidamente canalizadas en pro de lograr la respectiva inserción de los contenidos preestablecidos y esquematizados de acuerdo con las necesidades investigativas.

En función de lo planteado, se puede acotar que, para llegar a una Operacionalización del proceso investigativo, se considera pertinente el método sintético visto por Montemayor (2018) como aquel que "permite realiza una operación mental, mediante la cual se reduce la totalidad del tema de manera esencial”. (p.72). Es decir, su utilización hizo posible incluir apreciaciones descriptivas en función del problema para finalizar en un conjunto de inferencias que ayudan a caracterizar o sistematizar las informaciones obtenidas.

\section{Tipo de Investigación.}

La actividad de indagación se convierte en una práctica esencial para la selección de un nivel investigativo, momento en cuanto al grado de ofrecer los respectivos hechos de profundidad que el investigador busca darle al trabajo científico. Por eso las investigaciones documentales, son vistas desde la perspectiva de Montemayor (ob.cit) "es un escrito que trata de probar que algo es deseable caracterizada por la discusión y soluciones alternarles que generan conclusiones". (p.13)

En razón de la conceptualización citada, se puede entender que el objetivo trazado ubica al presente proceso científico en el campo documental. Por lo tanto, una vez seleccionado el tema se establecen las condiciones viables para la recolección de información significativa que permita desarrollar cada una de las características que identifican previamente al fenómeno en estudio.

\section{Fuentes Documentales.}

El desarrollo del proceso investigativo requiere una postura personal del investigador; razón por la cual, debe seleccionar las fuentes documentales encargadas de ofrecerle el respectivo apoyo 


\section{Procedimientos Adecuados para los Pacientes Intervenidos en Cirugía de Reasignación sexual}

Vol. 3, núm. 2., (2019)

Belforth Iván Vera Ganchozo; Diana Carolina Anchundia Molina; Jorge Luis Castillo Avendaño; Edith Elizabeth Cedeño Gavilánez; María José Saltos Loor; Dolly Natasha Ross Mera

para la elaboración del escrito. En tal sentido, Martínez (ob.cit) representa "un panorama acerca de la información relevante de diferentes fuentes confiables sobre un tema específico”. (p.33).

De este modo, para preparar el desarrollo de los diferentes contenidos teóricos propuestos, se hizo necesario la organización del material para su respectivo análisis, extracción e interpretación de aquellos aspectos relevantes e importantes para la construcción el artículo, en otras palabras, que guarden relación con el tema de los procedimientos adecuados para los pacientes intervenidos en cirugías de reasignación sexual, lo que ayuda a sintetizar las ideas y después son presentadas en el reporte final.

\section{Técnica de Recolección de Información.}

El análisis documental representa un trabajo caracterizado por la descripción de forma exhaustiva de todos los elementos presente en un documento. En el caso, particular de las investigaciones documentales es determinante el uso de técnicas interpretativas que ayuden a ampliar con lecturas críticas aquellas informaciones provenientes de fuentes específicas, sobre un tema para así llegar a las respectivas argumentaciones pertinentes.

Para encontrar la información sobre un tema en particular, se utilizan lecturas críticas, revisión de recursos tecnológicos a través de las diferentes páginas Web que poseen de forma accesible documentos importantes. Tal como lo indica Martínez (ob.cit) en la investigación documental las técnicas "ayudan a revisar, extraer, interpretar, analizar aspectos de interés para crear nuevas secciones". (p.34)

\section{Resultados.}

Con el fin de organizar los respectivos contenidos que estructuran al presente escrito, se fijaron como elementos subyacentes, una presentación particular para así llegar a la formulación de las consideraciones generales del mismo. Por ello, las apreciaciones logradas a lo largo del discurso, fueron trazadas desde una perspectiva particular, esto hizo posible su desarrollo en diferentes acotaciones para finalmente entender la significación que posee para los individuos una 


\section{Procedimientos Adecuados para los Pacientes Intervenidos en Cirugía de \\ Reasignación sexual}

Vol. 3, núm. 2., (2019)

Belforth Iván Vera Ganchozo; Diana Carolina Anchundia Molina; Jorge Luis Castillo Avendaño;

Edith Elizabeth Cedeño Gavilánez; María José Saltos Loor; Dolly Natasha Ross Mera

intervención de reasignación sexual, en otras palabras, su recorrido permitió elaborar los aspectos básicos que se fijan como parte esencial en el marco de cumplir los diferentes procedimientos adecuados encargados de dar cabida a la respectiva identificación sexual positiva para los seres humanos.

\section{Transexualidad.}

Las conductas de rol sexual caen en un continuo de masculinidad o feminidad tradicional, aunque culturalmente se reconoce cada vez más que algunas personas no se ajustan (ni se quieren ajustar necesariamente) a la dicotomía hombre-mujer tradicional. Estas personas pueden referirse a ellos mismos como de género diferente, no binarios o uno de muchos otros términos que se han vuelto más utilizados en los últimos 10 años.

En el campo de las culturas occidentales son más tolerantes con las conductas no conformistas de género (marimacho) en las niñas (lo que generalmente no se considera un trastorno de género) que con las conductas afeminadas o de mariquitas en los varones. Muchos varones juegan como niñas o actúan como sus madres, lo que incluye probarse la ropa de su hermana o su madre. En general este comportamiento es parte del desarrollo normal. La disconformidad de género (conducta que difiere de las normas culturales para el sexo de nacimiento de un individuo) en los niños no se considera un trastorno y rara vez persiste en la edad adulta o conduce a la disforia de género, aunque los niños que manifiestan disconformidad persistente pueden ser más propensos a eventualmente ser homosexuales o bisexuales en su adultez.

En este sentido, Rizo (ob.cit) precisa que para la mayoría de "las personas, hay congruencia entre sexo biológico (nacimiento), identidad de género y rol sexual. Sin embargo, los sujetos que tienen disforia de género experimentan cierto grado de incongruencia entre su sexo de nacimiento y su identidad sexual.” (p.29). De allí, que la incongruencia de género en sí no se considera un trastorno; sino la percepción o falta de correspondencia entre el sexo de nacimiento e identidad de género que se siente provoca malestar o discapacidad, un diagnóstico de disforia de género puede ser apropiado. 


\section{Procedimientos Adecuados para los Pacientes Intervenidos en Cirugía de Reasignación sexual}

Vol. 3, núm. 2., (2019)

Belforth Iván Vera Ganchozo; Diana Carolina Anchundia Molina; Jorge Luis Castillo Avendaño; Edith Elizabeth Cedeño Gavilánez; María José Saltos Loor; Dolly Natasha Ross Mera

Cabe destacar que, el malestar es típicamente una combinación de ansiedad, depresión e irritabilidad. Las personas con disforia de género grave, a menudo llamadas personas transexuales o transexuales, pueden experimentar síntomas severos, inquietantes y persistentes y tienen un fuerte deseo de una transformación médica y/o quirúrgica de su cuerpo para que esté más alineado a su identidad de género. En este particular, Butler (2015), destaca que, aunque "faltan cifras precisas, se estima que entre el 0,005 y el 0,014\% de los varones al nacer y el 0,002 el 0,003\% de las mujeres al nacer cumplen con los criterios diagnósticos de disforia de género". (p.33).

De este modo, se comprende que los factores biológicos como: dotación genética, medio hormonal prenatal determinan la identidad sexual, formación de una identidad sexual segura y sin conflictos y el rol sexual dependen también de factores sociales, como la relación emocional con los padres y la relación que cada uno de ellos tiene con el niño. Algunos estudios muestran una tasa de concordancia mayor para la disforia de género en los gemelos monocigóticos que en gemelos dicigóticos, lo que sugiere que existe un componente hereditario en la identidad transgénero.

Pocas veces la transexualidad se asocia con ambigüedad genital (afecciones intersexuales, trastornos del desarrollo sexual) o una anomalía genética como el llamado síndrome de Turner o síndrome de Klinefelter. Cuando la clasificación sexual y la situación en que se cría el niño son confusas (en casos de genitales ambiguos o síndromes genéticos que alteran el aspecto de los genitales, como los síndromes de insensibilidad a los andrógenos), los niños pueden no estar seguros sobre su identidad o su rol sexual, aunque el nivel de importancia de los factores ambientales sigue siendo controvertido. Sin embargo, cuando la clasificación sexual y la forma en que se cría el niño son claras, ni siquiera la presencia de genitales ambiguos puede afectar el desarrollo de su identidad sexual.

En relación a estos planteamientos, la disforia de género en la niñez suele manifestarse a los 2 a 3 años de edad. Usualmente los niños hacen lo siguiente: Prefieren vestirse como el otro sexo. Insisten en que pertenecen al otro sexo. Desean despertarse perteneciendo al otro sexo. 


\section{Procedimientos Adecuados para los Pacientes Intervenidos en Cirugía de \\ Reasignación sexual}

Vol. 3, núm. 2., (2019)

Belforth Iván Vera Ganchozo; Diana Carolina Anchundia Molina; Jorge Luis Castillo Avendaño;

Edith Elizabeth Cedeño Gavilánez; María José Saltos Loor; Dolly Natasha Ross Mera

Prefieren participar en juegos y actividades estereotípicos del otro sexo. Tienen sentimientos negativos sobre sus genitales. Por ejemplo, una niña puede insistir en que le crecerá un pene y se convertirá en un varón; puede intentar orinar de pie. Asimismo, el varón puede fantasear sobre ser mujer y evitar los juegos de manos y competencias. El varón puede sentarse para orinar y desear que le corten el pene y testículos. En los varones, la angustia por los cambios físicos de la pubertad a menudo es seguida por un pedido durante la adolescencia de tratamientos somáticos feminizantes. Tal como lo resalta Butler (ob.cit) "la mayoría de los niños con disforia de género no son evaluados hasta los 6 a 9 años, momento en el cual la disforia de género ya es crónica.” (p.33)

Cada uno de los planteamientos citados, llevan a destacar que la transexualidad es la adquisición por parte de una persona de las características físicas del sexo contrario mediante tratamiento hormonal o quirúrgico. La misma se presenta cuando una persona tiene una identidad de género distinta a la determinada por su cuerpo de nacimiento. Esta población frecuentemente soporta un fuerte estrés conocido como estrés de las minorías, debido a los habituales episodios de discriminación de los que son objeto, incluso agresiones físicas y verbales. Además, están las expectativas de discriminación generadas o actitudes de rechazo internalizado y aislamiento social por la falta de adaptación a la situación.

De lo antes indicado, se desprende lo citado por Verde (2016) destaca en cuanto a la discriminación que sufren los transexuales se puede indicar lo siguientes:

Un 81,75\% recibió algún tipo de agresión física a lo largo de la vida, el 31,16\% percibió discriminación laboral. El 25,89\% tuvo ansiedad social y un 22,84\% presentó al menos un intento de suicidio a lo largo de la vida. El factor más asociado con los síntomas depresivos fue la falta de integración en el ámbito laboral. (p.52)

Según lo planteado, se precisa que la actuación social y laboral, tiene una alta injerencia en la adaptación de este grupo etario, los mismos, como resultado a las actitudes que manifiestan otros individuos, los pueden llevar hasta el suicidio, por ello, es importante entender que la transexualidad se da cuando una persona tiene una identidad de género distinta a la determinada 


\section{Procedimientos Adecuados para los Pacientes Intervenidos en Cirugía de Reasignación sexual}

Vol. 3, núm. 2., (2019)

Belforth Iván Vera Ganchozo; Diana Carolina Anchundia Molina; Jorge Luis Castillo Avendaño; Edith Elizabeth Cedeño Gavilánez; María José Saltos Loor; Dolly Natasha Ross Mera

por su cuerpo de nacimiento, evento que los lleva a la discriminación contra las personas lesbianas, gais, bisexuales, trans e intersex. Pero, a pesar de los avances legales y sociales ocurridos en los últimos años, todavía esta población sufre altos niveles de discriminación. De allí, la necesidad de conocer las dificultades de tipo social y psicológico que presentan a fin de poder ayudar a diseñar mejores intervenciones y prevenir las causas de estas dificultades, por ello, la intervención quirúrgica de reasignación sexual, se ha convertido hoy en una alternativa efectiva para lograr en estas personas su aceptación personal.

\section{Cirugías de Reasignación Sexual.}

El concepto de reasignación de sexo se refiere a una intervención quirúrgica que permite modificar la genitalidad biológica de un ser humano para convertirla en una genitalidad acorde al género con el cual el sujeto se siente identificado. Esto quiere decir que el género asignado por naturaleza se reasigna mediante un procedimiento médico. Es decir, una persona que nace con género masculino siempre se sintió mujer. Después de consultar a psicólogos y otros especialistas, toma la decisión de someterse a una reasignación de sexo. De este modo, cuando finalice el proceso, pasará a tener genitales propios de una mujer, perdiendo aquello que la biología le había asignado.

En esta dirección, Rizo (ob.cit) define a la cirugía de reasignación de sexo es el nombre exacto que tiene esa intervención que "se realiza a personas en casos de intersexualidad, que es cuando se presenta una discrepancia entre sexo, genitales, y también a transexuales que quieren completar lo que es su cambio de sexo de una manera definitiva." (p.31). Es decir, es importante saber que hay pacientes que se someten a dicha intervención presentando más riesgos de que surjan complicaciones porque tienen obesidad, diabetes o problemas en lo que es la coagulación. De ahí que haya que extremar la atención y cuidados en su operación y también en el postoperatorio, ya que pueden sufrir dificultades con respecto a la anestesia e incluso con su recuperación.

De lo expuesto, se puede indicar que para la realización de esta intervención quirúrgica no sólo es los procedimientos postoperatorios, sino, aquellos valorados en el preoperatorios, dado que, su 


\section{Procedimientos Adecuados para los Pacientes Intervenidos en Cirugía de \\ Reasignación sexual}

Vol. 3, núm. 2., (2019)

Belforth Iván Vera Ganchozo; Diana Carolina Anchundia Molina; Jorge Luis Castillo Avendaño; Edith Elizabeth Cedeño Gavilánez; María José Saltos Loor; Dolly Natasha Ross Mera

conjugación garantiza su recuperación, por ello, es importante precisar que el paciente sea: mayor de edad, disponer de un certificado expedido por un psiquiatra que descarte cualquier tipo de alteración psicológica de tipo transitorio; pues, el proceso de reasignación de sexo tiene que ser tratado de manera integral porque si decides hacerlo se sufren cambios en múltiples aspectos: hormonal, psicológico, familiar, legal, social entre otros.

Es importante acotar que, para empezar a intervención de reasignación sexual se tiene que hacer una evaluación psicológica donde se confirma que se trata de un verdadero caso de transexualidad. Una vez que todo está claro el psicólogo le acompaña durante todo el proceso tanto al prospecto como a su familia. Luego, se empieza el tratamiento hormonal cruzado; hombres que se sienten mujeres son tratados con estrógenos y antiandrógenos y mujeres que se sienten hombres con andrógenos. Para así, cumplir un lapso de dos años identificado como la prueba de vida real, tiempo en el que deben de vivir con el género en el que se reconocen. Esto suele ser difícil pues, la transición está a medias y si la persona se arrepiente esto se tarda, pero puede revertirse. Pasado este tiempo lo que sigue es irreversible la cirugía.

En el caso de hombre a mujer transexual se realiza la penestomía y se retiran los testículos y se realiza una vaginoplastía y una vulvaplastía, además de implantes mamarios. A las mujeres que desean convertirse en hombres, se les retira el útero, ovarios y se extirpan los senos. Generalmente para construir el pene se extrae un injerto de piel del brazo o del muslo ayudado de una prótesis. El clítoris se deja en la base del pene. Esto no siempre es efectivo y lleva sus riesgos. Lo ideal de iniciar este proceso es en la adolescencia con el tratamiento que bloquea el proceso de desarrollo y este tratamiento es irreversible. Además de todo esto hay que contar con el tiempo, la paciencia, tolerancia a los cambios.

De lo antes expuesto, se puede indicar que la cirugía de resignación sexual es lo que más ha avanzado estos últimos 15 años en cirugía plástica y reconstructiva, según Mañero (2017) esta intervención "es toda aquella cirugía dedicada y diseñada para mejorar la vida de los transexuales que quieren hacer mejoras en su aspecto para identificarse mejor en el mundo". (p.8). Por ello, mediante esta intervención quirúrgica, los transgénero por ser aquella persona que 


\section{Procedimientos Adecuados para los Pacientes Intervenidos en Cirugía de Reasignación sexual}

Vol. 3, núm. 2., (2019)

Belforth Iván Vera Ganchozo; Diana Carolina Anchundia Molina; Jorge Luis Castillo Avendaño; Edith Elizabeth Cedeño Gavilánez; María José Saltos Loor; Dolly Natasha Ross Mera

no se siente identificado con el género que sociedad le ha impuesto según su sexo biológico, es decir, cuando nace siendo mujer biológicamente, pero sus ideales son masculinas. Una persona transgénero puede tener diferentes orientaciones sexuales como: heterosexual, homosexual, bisexual.

En este orden de ideas, la cirugía de resignación de sexo también conocida como confirmación de género o cirugía de resignación sexual, está siendo cada vez más demanda y aceptada en la sociedad, esto se debe a la normalización de la transexualidad en muchos ámbitos, también es relevante en el campo de la cirugía plástica su avance ha crecido en el tiempo haciéndose cada vez menos traumática.

\section{Figura $\mathbf{N}^{\circ} 1$ Reasignación Sexual}

Fuente: Mañero (ob.cit)

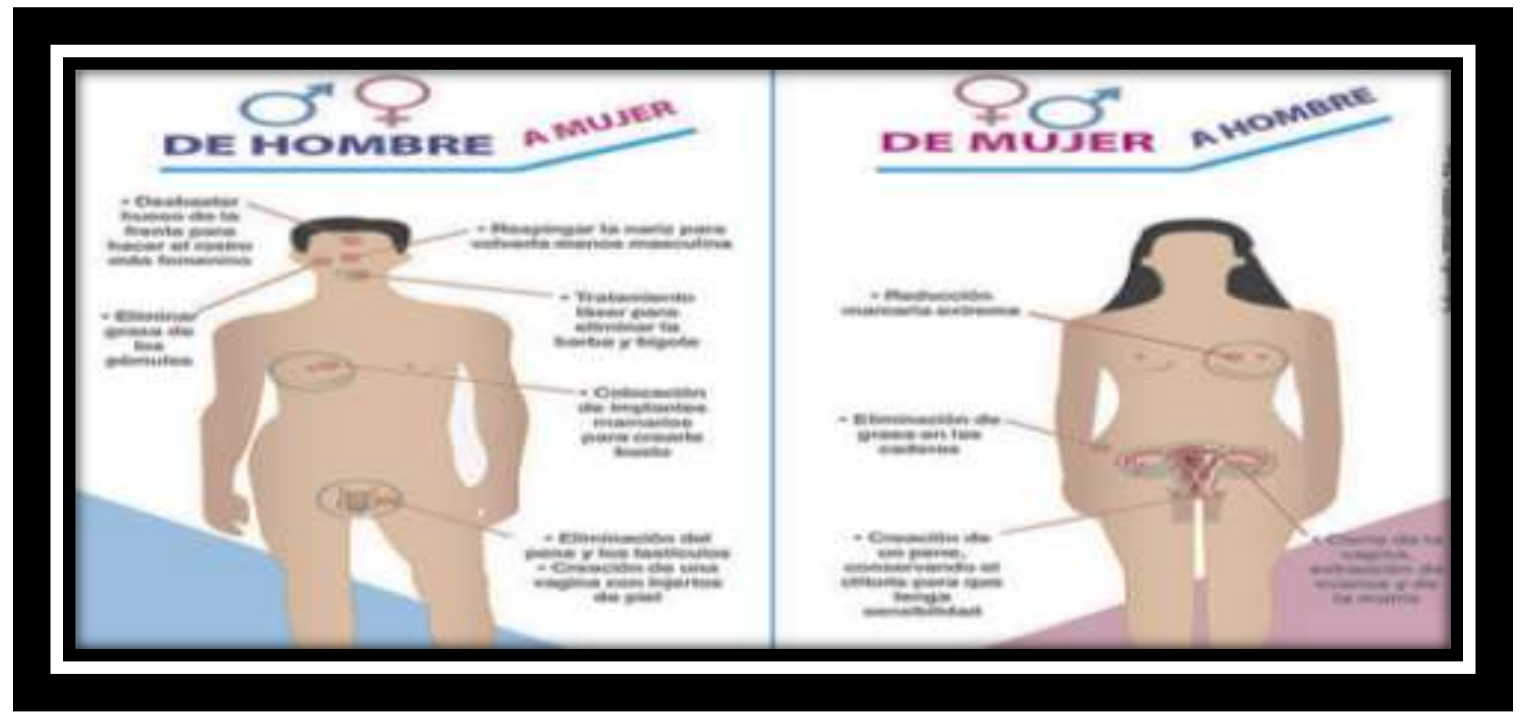

Cirugía de Transexualidad Femenina: afecta a las personas que tienen sexo masculino, pero, se consideran mujer tienen una identidad de género femenino, en este caso la cirugía se denomina genitoplastía, la vaginoplastía por inversión peneana. El cirujano invierte la piel del pene y el escroto con la finalidad de crear una vagina, tanto a nivel estético como funcional lo más femenina posible. También puede realizarse una vaginoplastía con colgajo rectosigmoidal pediculado este proceso consiste en utilizar la parte final del intestino para crear la vagina. El 


\section{Procedimientos Adecuados para los Pacientes Intervenidos en Cirugía de \\ Reasignación sexual}

Vol. 3, núm. 2., (2019)

Belforth Iván Vera Ganchozo; Diana Carolina Anchundia Molina; Jorge Luis Castillo Avendaño; Edith Elizabeth Cedeño Gavilánez; María José Saltos Loor; Dolly Natasha Ross Mera

cirujano determinará cual es la técnica más adecuada dependiendo de las características concretas del paciente. Asimismo, se realizan las cirugías estéticas feminización tanto en la cara (rinoplatia Lifting Frontal Mentoplastia, como en el cuerpo principalmente Mamoplastia y Lipoescultura

Cirugía de Transexualidad Masculina: Las personas que nacen con sexo femenino, pero se identifican con el sexo masculino. La Metaidoiplastia es la cirugía que posibilita la construcción de un pene a partir del clítoris previamente tratado hormonal para conseguir su hipertrofia. Se consigue dotar al paciente con un pene pequeño con la mayor parte de su funcionalidad; pero que no apto para llevar a cabo la penetración sexual Otra técnica es la conocida como Faloplastía que a diferencia de la anterior el pene es construido con tejido de otras partes del cuerpo como piel del antebrazo que posibilita una mayor longitud. Requiere la implantación de prótesis testiculares en una segunda intervención y según el caso prótesis perneana También son habituales cirugías Estéticas Masculizantes faciales y corporales.

No obstante, Mañero (ob.cit) precisa que la cirugía de reasignación de sexo tiene un alto riesgo de complicaciones. Las más graves tienden a concentrarse en las primeras 48 horas. Solo el $60 \%$ de los pacientes logran sensibilidad en el pene. El 30\% sufre una pérdida total o parcial del falo. El peligro de sufrir cáncer de mama aumenta, pese a las mastectomías, debido al tratamiento hormonal. Y normalmente es necesario colocar, además, una prótesis en el nuevo miembro.

Este mismo autor, acota que la intervención es irreversible. Son pocos los casos en que los pacientes piden volver al estado anterior, un $0,6 \%$, los resultados no son buenos. Por lo tanto, se recomienda al paciente y familiares acudir a asociaciones, con el fin de conocer personas que han vivido lo mismo. Y también que pasen previamente por un periodo de experiencia de vida real; que vivan en el cuerpo que sienten, cuando se sientan confortables, para poder continuar.

Otro aporte de importancia para la realización de la intervención de reasignación sexual, es lo indicado por Mañero (ob.cit) el llamado método Fakin basado en el cuidado integral de cada paciente, desde una perspectiva completa de su realidad. El equipo multidisciplinar experto en cirugía plástica y estética, es especialista sobre todo en pecho, facial y cuello, así como contorno 


\section{Procedimientos Adecuados para los Pacientes Intervenidos en Cirugía de Reasignación sexual}

Vol. 3, núm. 2., (2019)

Belforth Iván Vera Ganchozo; Diana Carolina Anchundia Molina; Jorge Luis Castillo Avendaño; Edith Elizabeth Cedeño Gavilánez; María José Saltos Loor; Dolly Natasha Ross Mera

corporal. Mediante este método cada vez es menos invasivos y más personalizadas las intervenciones, se usa la combinación de varias técnicas vanguardistas, innovadoras y ampliamente probadas, que permite resultados más naturales, atractivos y una recuperación rápida y segura.

Asimismo, precisa que mediante la combinación de técnicas ha permitido comprobar los beneficios de esta técnica que proporciona una sensibilidad 100\%, además de apariencia y funcionalidad óptimas. El resultado permite tener relaciones sexuales satisfactorias, ponerse cualquier tipo de ropa y llevar la vida normal a las transexuales. La técnica quirúrgica permite, en una sola intervención, realizar la reasignación genital y el aumento de pechos, no siendo necesaria una segunda cirugía meses después, que es el caso en los sistemas tradicionales. El porcentaje de complicaciones en las cirugías de reasignación de género realizadas por el referido método es de menos del $3 \%$ y todas de menor importancia, mientras que al comparar con el método tradicional existe un índice del 33\% de complicaciones en muchos casos de importancia.

De lo citado anteriormente, se encuentra una vez realizada la respectiva intervención de reasignación sexual, unos significativos procedimientos con el fin de lograr la respectiva recuperación del paciente, los mismos son destacados por Montilla (2019), entre los cuales se encuentran los siguientes:

El o la paciente debe permanecer hospitalizada por un lapso de siete días, después de la intervención, con el fin de ser valorada diariamente por el equipo médico.

Se evaluarán mediante un protocolo de caso los signos vitales, realización de un aumento progresivo de la dieta, rotación de analgesia y antibióticos por vía oral, deambulación precoz y buen manejo del dolor.

El cirujano en caso del cambio hacia el sexo femenino, se encargará de orientar a la paciente en cuanto al mecanismo de dilatación vaginal, el cual debe cumplir diariamente para asegurar con ello una respuesta sexual oportuna al momento del ejercicio de la función sexual. 


\section{Procedimientos Adecuados para los Pacientes Intervenidos en Cirugía de \\ Reasignación sexual}

Vol. 3, núm. 2., (2019)

Belforth Iván Vera Ganchozo; Diana Carolina Anchundia Molina; Jorge Luis Castillo Avendaño;

Edith Elizabeth Cedeño Gavilánez; María José Saltos Loor; Dolly Natasha Ross Mera

Asimismo, se evaluará la contención social que el grupo familiar presenta con el paciente, además de revisar su vulnerabilidad ante los cambios físicos que observe y pueda generar algunas situaciones negativas.

Revisión del proceso de cicatrización, para facilitar posteriormente la sensibilidad sexual, Otro procedimiento la asignación del tratamiento instaurado de analgesia y antibioticoterapia. Una vez en casa, la paciente deberá ponerse en contacto con el endocrinólogo que supervisa su tratamiento hormonal para reiniciarlo (pasado unas 4 semanas) y reajustar la dosis. En ningún caso, la paciente reajustará por sí misma las dosis de hormonación ya que esto podría suponerle problemas de salud.

En el postoperatorio tardío pueden aparecer estenosis vaginal o del introito; estenosis uretral o del meato; fístula recto-vaginal; sufrimiento del clítoris; trombosis venosa o embolias. Un miembro del equipo médico indicará a la paciente cómo evitar estas posibles complicaciones y cómo actuar en el supuesto de que aparecieran. Se mantiene la sonda vesical hasta que la inflamación periuretral disminuya.

También, se harán revisiones permanentes en cuanto a los procesos de coagulación, dado que el consumo de estrógenos aumenta el riesgo de padecer trombos en sangre, de allí, la importancia de vigilar periódicamente estos procesos a fin de reducir los efectos negativos de los coágulos de sangre en el interior de las venas. De igual manera, se recomienda hacer evaluaciones permanentes para evaluar otras enfermedades como cáncer debido al consumo de las hormonas.

\section{Conclusiones.}

Los aspectos desarrollados llevan a destacar a continuación una serie de conclusiones generales, que permiten ofrecer las reflexiones dadas por el investigador a fin de lograr la respectiva valoración del tema tratado. Entre ellas se citan las siguientes:

Según los aportes teóricos, se precisa que las personas con sexo biológico masculino, esta cirugía implica la extirpación de parte del pene y de los testículos y la creación de una vagina artificial. 


\section{Procedimientos Adecuados para los Pacientes Intervenidos en Cirugía de Reasignación sexual}

Vol. 3, núm. 2., (2019)

Belforth Iván Vera Ganchozo; Diana Carolina Anchundia Molina; Jorge Luis Castillo Avendaño; Edith Elizabeth Cedeño Gavilánez; María José Saltos Loor; Dolly Natasha Ross Mera

La parte del pene que se deja actúa como un clítoris, ya que suele ser sexualmente sensible y posibilita el orgasmo. En las personas con sexo biológico femenino, supone la extracción quirúrgica de las mamas (mastectomía) y en ocasiones de los órganos reproductores internos (útero y ovarios), el cierre de la vagina y la creación de un pene artificial. Los resultados de la cirugía de mujer a hombre son menos satisfactorios que los de la cirugía de hombre a mujer en cuanto a la apariencia y funcionalidad, lo que posiblemente explica por qué un menor número de transexuales femeninos solicitan la cirugía de reasignación de sexo

Por ello, la realización de una intervención de reasignación sexual, debe existir un equipo multidisciplinario, encargado de evaluar las condiciones psicológicas, físicas, fisiológicas, sociales y familiares del paciente, pues, cada una de ellas tiene injerencia en sus actitudes y son los encargados de proporcionar el éxito de la misma. Según, los aportes teóricos, los datos arrojados en cuanto a la negación del proceso quirúrgico porcentualmente es reducido, esto permite entender que los transexuales debido a la no aceptación de su sexo biológico, buscan mediante dicha actividad lograr el respectivo cambio que desean como persona.

Durante la realización de la cirugía de reasignación sexual, se evidencia que se originan cambios en los órganos externos e internos, como el caso de las mujeres que su útero, ovarios, trompa de Falopio son extirpados, y en los hombres para convertirlos en mujeres se introduce un proceso de aumento de las mamas. Asimismo, se puede indicar, que los progresos científicos originados a nivel de las ciencias médicas llevan a considerar dicho proceso quirúrgico como un adelanto de importancia para las personas transexuales, reconociendo con ello, su derecho a tener una vida psicológica saludable que reduzca los efectos de la discriminación.

Cabe destacar que, los procedimientos adecuados una vez efectuada la intervención quirúrgica, se convierten en elementos esenciales para garantizar resultados favorables, por ello, el o la paciente, deben cumplir a cabalidad los lineamientos dados, mediante una actuación asertiva, se logra su respectiva reincorporación a la vida social, laboral, familiar y además se garantiza un bienestar psicológico, como resultado al incremento de sus niveles de autoestima, al dejar a un lado la percepción de su propio sexo biológico. 


\section{Procedimientos Adecuados para los Pacientes Intervenidos en Cirugía de \\ Reasignación sexual}

Vol. 3, núm. 2., (2019)

Belforth Iván Vera Ganchozo; Diana Carolina Anchundia Molina; Jorge Luis Castillo Avendaño;

Edith Elizabeth Cedeño Gavilánez; María José Saltos Loor; Dolly Natasha Ross Mera

\section{Bibliografía.}

Butler, J. (2015). Cambio de Sexo en Personas Transgénero. Infosalud, 52-59.

Mañero, I. (2017). Reasignación Sexual . IMCline , 7-12.

Martínez, A. (2018). El Método de Investigación . Madrid : Morata .

Montemayor, M. (2018). Documentos para la Investigación Documental . México: Trillas .

Montilla, C. (2019). La Cirugía de Reasignación Sexual . Infosalud , 3-12.

Rizo, D. (2015). Aceptación o Resignación Sexual . Las Mentes Maravillosas , 28-37.

Ruiz, M. (2015). La Reasignación una peligrosa Comodidad . Psicología y Terapia , 14-26.

Verde, M. (2016). Hacerle Justicia a alguien: la reasignación de sexo y las alegrías de la transexualidad. ELSEVIER, 32-41.

$$
\text { (9) (1) } \Theta(0
$$

RECONOCIMIENTO-NOCOMERCIAL-COMPARTIRIGUAL

CC BY-NC-SA

ESTA LICENCIA PERMITE A OTROS ENTREMEZCLAR, AJUSTAR Y CONSTRUIR A PARTIR DE SU OBRA CON FINES NO COMERCIALES, SIEMPRE Y CUANDO LE RECONOZCAN LA AUTORÍA Y SUS NUEVAS CREACIONES ESTÉN BAJO UNA LICENCIA CON LOS MISMOS TÉRMINOS. 